The workflow adopted for the FlexMaps Pavilion (Fig. 1) uses a fully automated approach for the definition and the control of the mesostructures (base geometry) and consequently of the material behavior. In the present work, an existing optimization method [10] is being utilized in a novel application domain of architectural-scale objects. Similar to other architectural-scale bending-active methods $[8,11]$, the present method allows for controlling the shape by means of a numerical form-finding. Hence, the load-bearing elements are altered in search for their best configuration, so that once assembled the obtained shape results as close as possible to an input target geometry. This is a step towards a designer/artist-oriented tool.

Compared to other architectural methods used for bending-active structures, another novelty of the present approach lies in the assembly. In post-restrained structures where the components are bent and fixed to the ground, a common approach is to post-tension the overall shape that initially lies in the flat position. This procedure requires external facilities, a large work area and usually requires the whole shape to be developable. Conversely, the FlexMaps approach is based on bending and assembling the single mesostructured element, which lays flat in the rest configuration. Eventually, the final shape of the structure results from internal elastic forces that redistribute among the elements once they are all connected. The construction sequence consists in bending the panels, one at a time, and progressively connecting them to the neighboring ones. This procedure has the advantage of requiring only a minimal amount of bending energy and can be performed by hand. The two cost-saving characteristics of the FlexMaps Pavilion originate from the easy fabrication that is obtained by producing a flat segmented array of panels, and from the simple assembly procedures that does not require any specialized manpower nor other tools. In FlexMaps examples, active-bending should be regarded in a broader sense of either bending and torsion deformations.

The main issue of bending-active structures is that the elastic bending causes initial stress. This lowers the stress reserves that the structure may attain due to external loading. The spiral geometry, which is the main idea behind the present project, tackles this point. Having a spiral path instead of a linear path brings to minor bending stress for the same curvature. This phenomenon can be well observed from the example in Fig. 2 , in which it is reported the stress obtained through FEA for three different panels that are deformed with the same given curvature.

Moreover, the spiraling geometry can be favorably modified to obtain bespoke FlexMaps panels in order to accommodate local curvature demands. A modification in the geometry consequently varies the bending stiffness, which in turn affects the bent shape, since all the panels tend to preserve a uniform stress once assembled. This approach corresponds to designing custom mechanical properties without changing the material but only by acting on the geometric parameters of spirals.

The objective of this work is to apply and validate the algorithm [10] for architectural design intents. The opportunity of testing the feasibility and extending this work from small objects to large scale objects has been provided by the call for "Competition and exhibition of innovative lightweight structures" organized in 2019 by the IASS Working Group 21 "Advanced manufacturing and materials" [12]. The competition posed as a limit for the experimental structure to fit a maximum volume of $4.00 \times 4.00 \times 4.00$ meters. Moreover, all the components should have fit into a maximum of six boxes up to $1.00 \times 0.75 \times 0.65 \mathrm{~m}$ and $32 \mathrm{~kg}$ each in order to be transportable by airplane.
Fig. 1 Photos of the FlexMaps Pavilion at the Competition and exhibition of innovative lightweight structures, Form and Force, joint international conference of IASS Symposium 2019 and Structural Membranes 2019
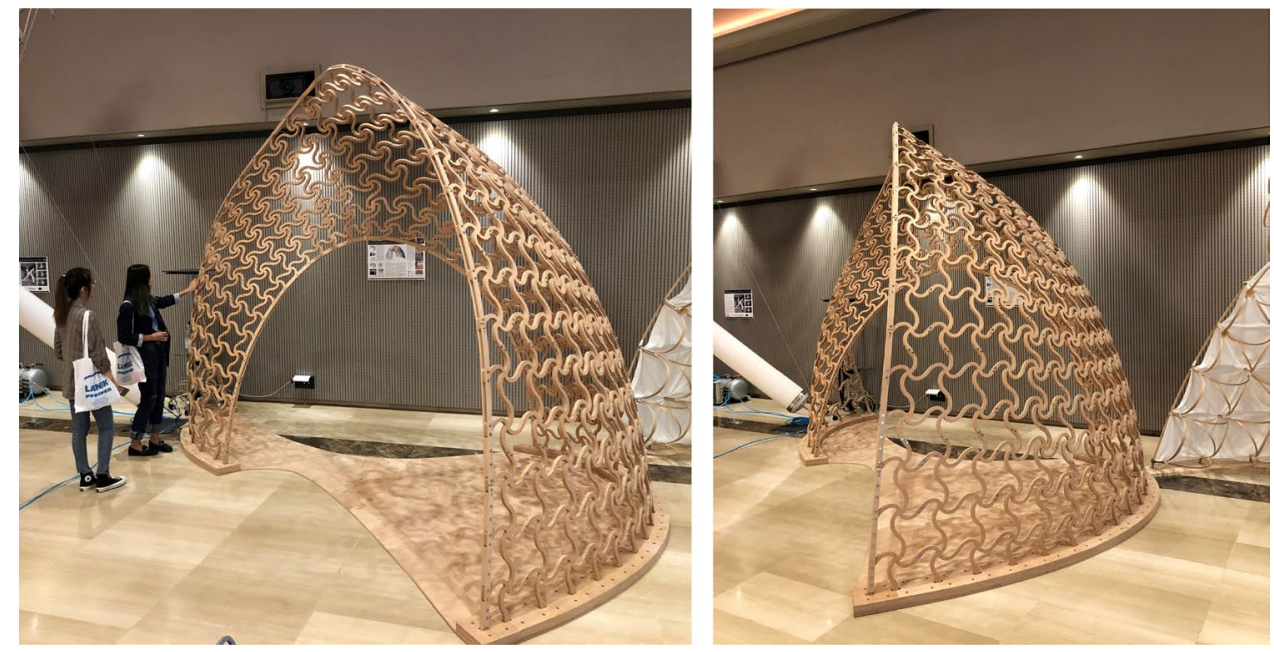
(a) A: Static Structural
Equivalent Stress
Type: Equivalent (vo Type: Equivalent (von-Mises) Stress Unit: $\mathrm{MPa}$ Time: 1 Time: 1 Custom Max: 1304.1 7/17/2020 4:04 PM

1304.1 500 437.5 $-375$ 312.5 250 187.5 62.5 0
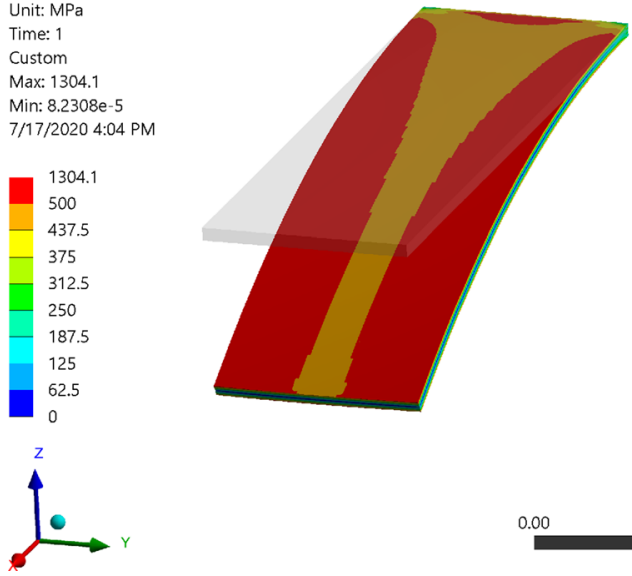

(b) (c)
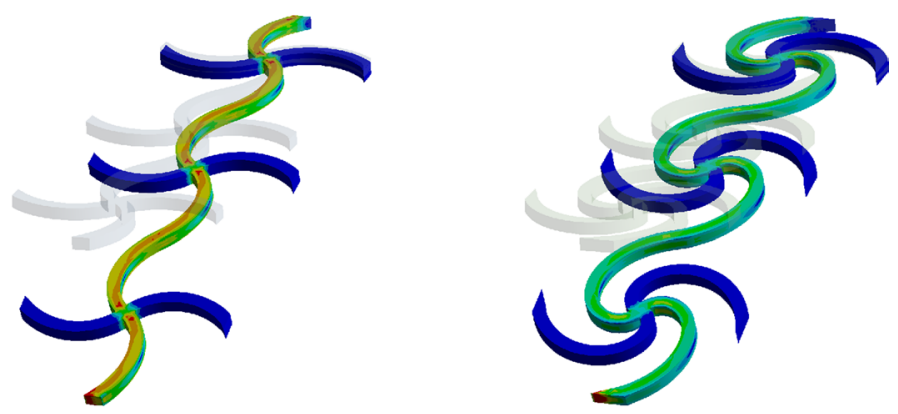

Fig. 2 Stress comparison of three bent plywood strips with same bounding box of $250 \times 750 \times 15 \mathrm{~mm}$, given a Y-rotation of $15^{\circ}(2 x$ displacement amplification): a solid plate (reactive $\mathrm{Y}$-moment:

We designed a non-trivial shape of a non-developable twisted arch with varying curvature demand, which has been later segmented in patches of the dimensions required for shipping. Special attention has been given to the plywood material selection, whose properties have been used to inform the reduced model that is embedded in the optimization routine, and for a subsequent FE validation of the design. In this pipeline, this latter step is fundamental to verify the real structural behavior of the Pavilion and to check its safety.

\section{FlexMaps concept}

The technique presented in [10] is designed to physically approximate an input 3D shape by interlocking a set of fabricated flat panels which are coupled solely by snapping together connectors on their boundaries. Following Flexmaps [10], a generic input shape is split into multiple panels which are then flattened onto a 2D plane. Each panel is made flexible by carving out long thin spiralshaped structures. The patterns have to be relatively dense and regular enough to be aesthetically pleasing once they are assembled to complete the final shape.

Inspired by the beauty of geometric tilings, FlexMaps uses a quadrilateral arrangement of spatially-varying, fourarm spiraling shapes. The spirals have sufficient degrees of freedom to be continuously shaped into multiple configurations by varying three primary parameters: scale, twist, and width. Some settings of the spiral are shown in Fig. 3. As it is possible to observe, the shape can undergo
$-4.88 \mathrm{kNm}$ ), b spiral with $50^{\circ}$ twist (reactive Y-moment: $-0.25 \mathrm{kNm}$ ), c spiral with $107^{\circ}$ twist (reactive Y-moment: $-0.14 \mathrm{kNm}$ )

a significant change of form, and every configuration corresponds to a particular set of mechanical properties.

This strategy follows a new philosophy of mechanical design of elastic properties that have been recently pursued by several optimization techniques [13]. Specific micro-scale structures can control a large variety of mechanical properties. Similarly, distributing different mesostructures on surfaces makes it possible to manufacture deformable shells with continuously varying mechanical properties.

In the case of FlexMaps the mechanical properties are optimized such that, once the structure is assembled, its static equilibrium configuration matches as much as possible the desired 3D shape. However, the final shape mainly depends on two main factors:

- Primitive Distribution. The panel subdivision and the distribution of the quads that embed the spiral has a great influence on the global equilibrium position of the entire FlexMap.

- Shape of the Spirals. The mechanical properties of the FlexMap can be controlled on a local basis by the varying shape of the spirals. While the underlying quad tessellation fixes the scale, twist and width can be adapted to match the desired flexibility.

The central core of the optimization process is a datadriven model that can predict the resulting deformed shape in 3D iteratively. The mechanical model is based on the rod coupling formulation in [14] and allows for approximating the range of spiral deformations with sufficient accuracy at an interactive rate. This model 
Fig. 3 Descriptors determining the geometry of our spiralshaped mesostructrures (left) and illustrative parameters exploration (right) [10]: the spirals can smoothly variate the twist (vertical axis) and width (horizontal axis)
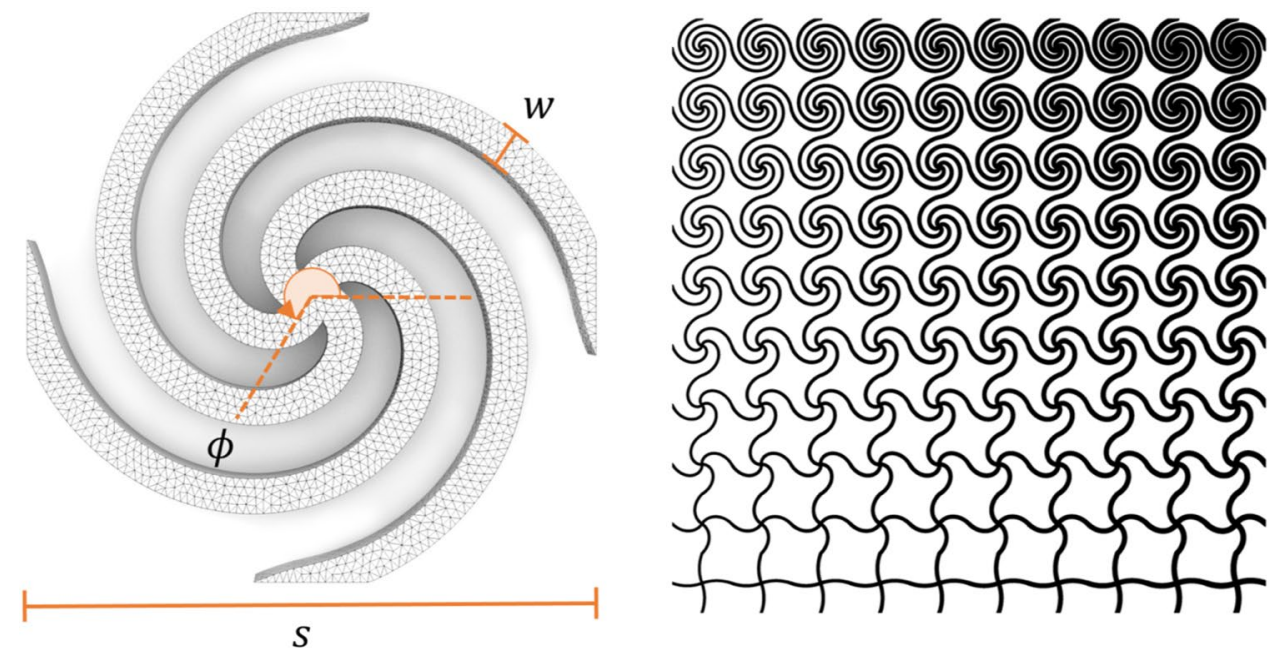

is based on a linear elastic material formulation, and uses the physical properties of the material utilized for fabrication.

The panel layout is initially derived using a state of the art algorithm [15], and then it is iteratively optimized by merging adjacent panels. This iterative optimization process tries to minimize the difference between the target shape and the predicted equilibrium shape computed by the physical model.

FlexMaps enables the design of elastic surfaces with heterogeneous material properties, by optimizing the parameters of the spirals to eventually match the target shape at the equilibrium configuration. Intuitively, regions of high curvature of the mesh should be populated with characteristically compliant spirals. However, the equilibrium configuration is the global result of all the mechanical properties of the spirals and the boundary conditions they must satisfy. The optimization of this objective is a complex iterative process that minimizes non-linear energies and that matches a set of linear constraints to ensure the shape to be in equilibrium (details of this process are in [10]). An overview of the entire processing pipeline is shown in Fig. 4.

\section{Design of the FlexMaps pavilion}

The FlexMaps Pavilion is a twisted lattice surface that is anchored on the ground. Base sections are inward-bent arches. The shape of a twisted arch has been selected to be a challenge in both geometric and architectural terms. Moreover, the shape delimits a space but also allows people to walk through it. There is a sufficiently-wide curvature range and no symmetry. The dimension of the bounding box is $3.90 \times 3.96 \times 3.25$ meters.

It is composed of several panels that can be deformed in bending and torsion and are mutually interlocked. Once the Pavilion is anchored to the ground, its final shape rises from internal stress. The bending and stretching resistance offered by each panel is optimized by tuning the geometry of the individual spirals (Fig. 5).

\subsection{Form finding and segmentation}

The twisted shape has been modeled manually. Then, it has been re-meshed with a quadrilateral meshing following the approach based on global parametrization proposed in [16]. The final aim is to obtain as regular as

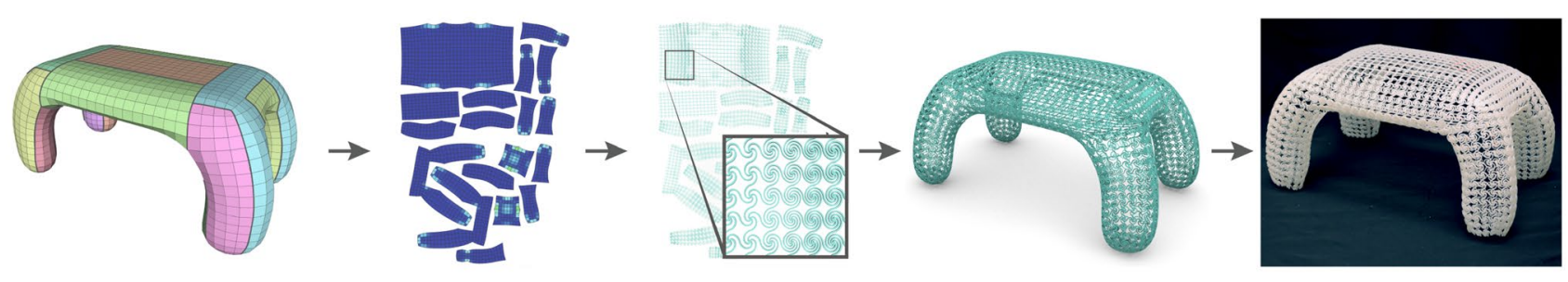

Fig. 4 An overview of Flexmaps pipeline [10]: an input mesh is split into multiple patches composed by quads; the patches are mapped on a 2D domain; a special spiral-shaped mesostructure is embed- ded on each quad; the parameters defining the shape of spiral mesostructure are optimized to improve accuracy in the final representation

\section{SN Applied Sciences}




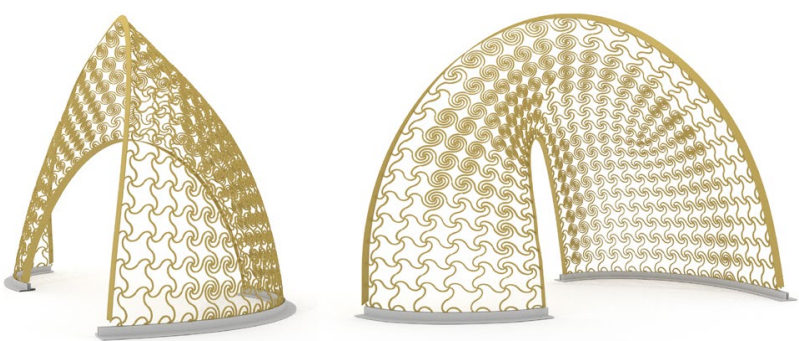

Fig. 5 Preliminary design overall view of the assembled plywood FlexMaps Pavilion. The metal base profile has been later replaced by an all-plywood base in the definitive design. Additionally the twist range of the spirals has been restricted to accommodate milling tolerances

possible quads with bounded dimension. The initial quad meshing is an essential step in the overall modeling pipeline since it defines the dimension of the spirals. In fact, each quad embeds exactly one spiral. The initial cross-field that drives the parametrization is aligned with the boundaries and is smoothly interpolated in the shape interior. Then, the shape of each quad is further optimized using the approach proposed in [17]. This optimization step is fundamental to ensure each spiral to be embedded in a regular quad. In case this is not verified, significant non-rigid deformations might introduce an unexpected mechanical behavior in the embedded spiral.

Due to fabrication constraints, target quad edges of $0.25 \mathrm{~m}$ have been selected. Moreover, for aesthetic reasons a constant width of $20 \mathrm{~mm}$ and a twist range of $100-250^{\circ}$ are chosen as input parameters. The upper twist limit has a further aim to avoid too dense spirals, which may result too close with respect to the milling tolerance. These restrictions are equivalent to a reduction of the design space for the optimization problem.

The generation of patches that cluster two or more quads can be automatically managed by the algorithm
FlexMaps or can be user-defined through a graphical user interface (details are in [10]). In the specific case of the Pavilion the quad mesh segmentation has to produce patches, which have to comply with the competition constraints of fitting six boxes, each with a maximum external size of $1 \times 0.75 \times 0.65 \mathrm{~m}$ and maximum weight of $32 \mathrm{~kg}$. Therefore, user-defined seams have been introduced each time a patch exceeded the box size. Finally, a total amount of 75 panels are generated, each grouping two up to six spirals (Fig. 6).

\subsection{Materialization}

The 2D FlexMaps panels are machine-milled from Okumé plywood panels. Since the forming principle of FlexMaps structures is the active-bending, the material has been selected according to the indications given by [18] and [1]. Particular attention has been dedicated to the recommended limitation $\sigma_{M, R d} / E>2.5 \mathrm{MPa} / \mathrm{GPa}$ for activelybent structures, in which $\sigma_{M, R d}$ is the bending strength of the material and $E$ is the Young's modulus. In this case, by inputting the data provided by the manufacturer (Table 1), this limitation equals $46 / 4.41=10.43 \mathrm{MPa} / \mathrm{GPa}$. By observing this fundamental limitation, the use of materials that can not attain high breaking strain during the bending process is avoided.

The ground edges are clamped in a CNC cut plywood profile; moreover, they are mutually connected and tied down. The naked edges that form two crossed arches are

Table 1 Okumé plywood material properties (technical data from the manufacturer)

\begin{tabular}{lllll}
\hline $\begin{array}{l}\text { Nominal } \\
\text { thickness } \\
(\mathrm{mm})\end{array}$ & N. Strata & $\begin{array}{l}\text { Density } \rho \\
\left(\mathrm{kg} / \mathrm{m}^{3}\right)\end{array}$ & $\begin{array}{l}\text { Young's } \\
\text { modulus } E \\
(\mathrm{MPa})\end{array}$ & $\begin{array}{l}\text { Bending } \\
\text { strength } \sigma_{M, R d} \\
(\mathrm{MPa})\end{array}$ \\
\hline 20 & 11 & 500 & 4410 & 46 \\
\hline
\end{tabular}
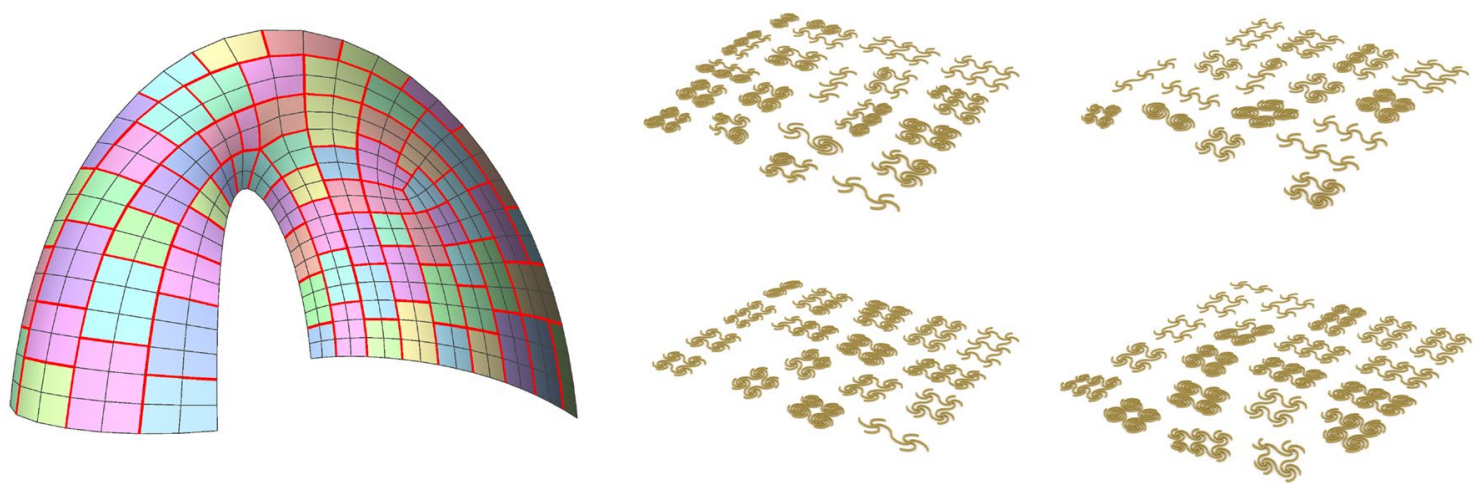

Fig. 6 Pavilion patches: schematic view of the Pavilion highlighting the seams and the initial quad mesh; preliminary-design of flat spiraling FlexMaps panels 
stiffened using a segmented machine-milled 6-mm plywood plate. The role of these arches is to provide additional restraint to the panels in the assembly phase. These arches have not been included in the optimization phase, i.e. as additional constraints, because of their low stiffness, which is mainly relevant in the in-plane direction. To connect FlexMaps together special connectors made of interlocking shapes, additionally secured by screws, are adopted.

\section{FE simulation}

For the detailed deformation and stress verification of the Pavilion a geometrically nonlinear solid model has been built and analyzed with the ANSYS package [19]. A linear elastic isotropic material model has been used in the analyses. These properties are obtained from material testing on plywood specimens.

The verification consists of two phases: the first one to model the individual assembly of the panels and their initial stress; the second one to model the overall behaviour under external loading, such as gravity.

The meshing used in the FE analyses is generated from a NURBS solid model, which is obtained by extruding the outline of the initial mesh.

In the first phase, each flat panel is rigidly moved in a position that is as close as possible to its deformed shape. This placement aims at improving the convergence of the analysis. The deformed shape is deduced from the form-finding phase. Then, a displacement is imposed at the extremities of the spirals' free arms to bring them in their deformed position. Consequently the FlexMaps panel assumes its pre-stressed configuration (Fig. 7a).

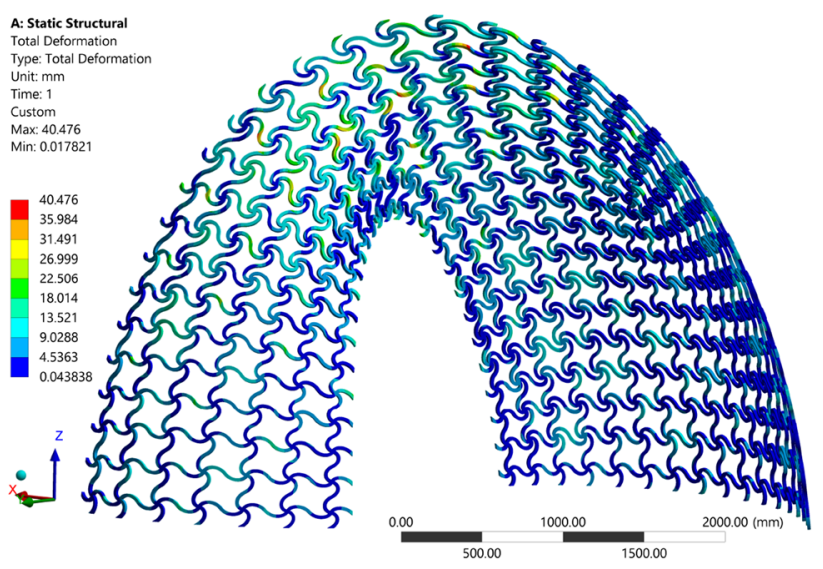

(a)
The individually deformed (pre-stressed) panels and the accompanying stress fields are exported in a second environment in which the panels extremes are coupled, and boundary conditions are applied. In this setup, the structure is released and gravity is applied (Fig. 7b). In this setting, other scenarios can be evaluated. However, since the pavilion has been designed as indoor installation no other loads have been considered.

As a result, the Pavilion exhibited a uniform redistribution of the stress due to the bending process. Moreover, the maximum strength of the material has not been exceeded not even in the loaded structure, in which peaks of $20-25 \mathrm{MPa}$ have been reached. The Pavilion exhibits also good stiffness and results close enough to the target shape. Indeed, the displacement with respect to the optimized target configuration is $38 \mathrm{~mm}$. In order to verify the safety level, the structure has been additionally checked for instability through buckling analysis.

\section{Fabrication and assembly}

The FlexMaps panels are CNC cut in the shop by means of double precision mill to accommodate tolerances of the interlocking shapes of connectors. The connectors have been previously tested in a four point bending setup to obtain a quantitative estimation of the stiffness reduction caused by the connection.

The Pavilion is assembled section by section going from one support to the other, sequentially bending and fastening each FlexMaps pane (Fig. 8). Moreover, these patches are spliced in a staggered configuration to minimize the bending energy that is required to keep the Pavilion section in place. To this purpose, the crossed arches play a fundamental

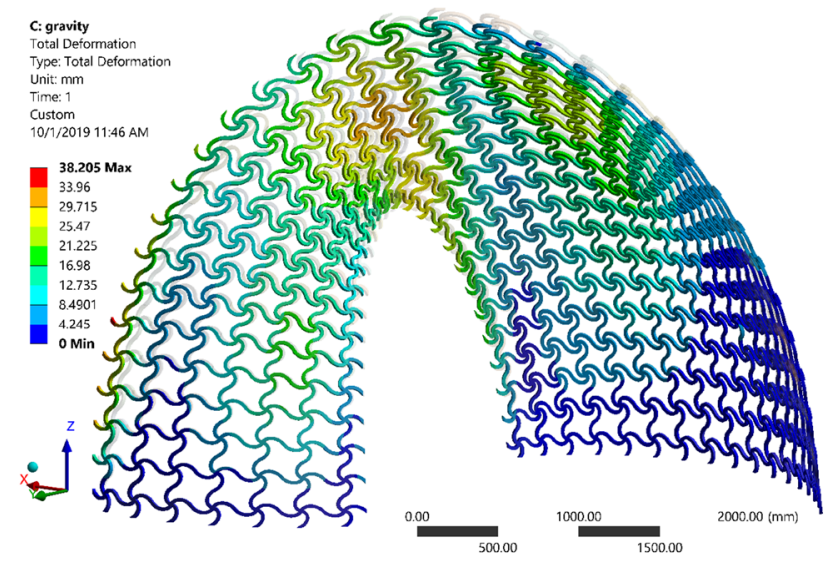

(b)

Fig. 7 FE analysis results: a phase 1 displacement, b phase 2 displacement under gravity load 
Fig. 8 Fabrication and assembly of the FlexMaps Pavilion
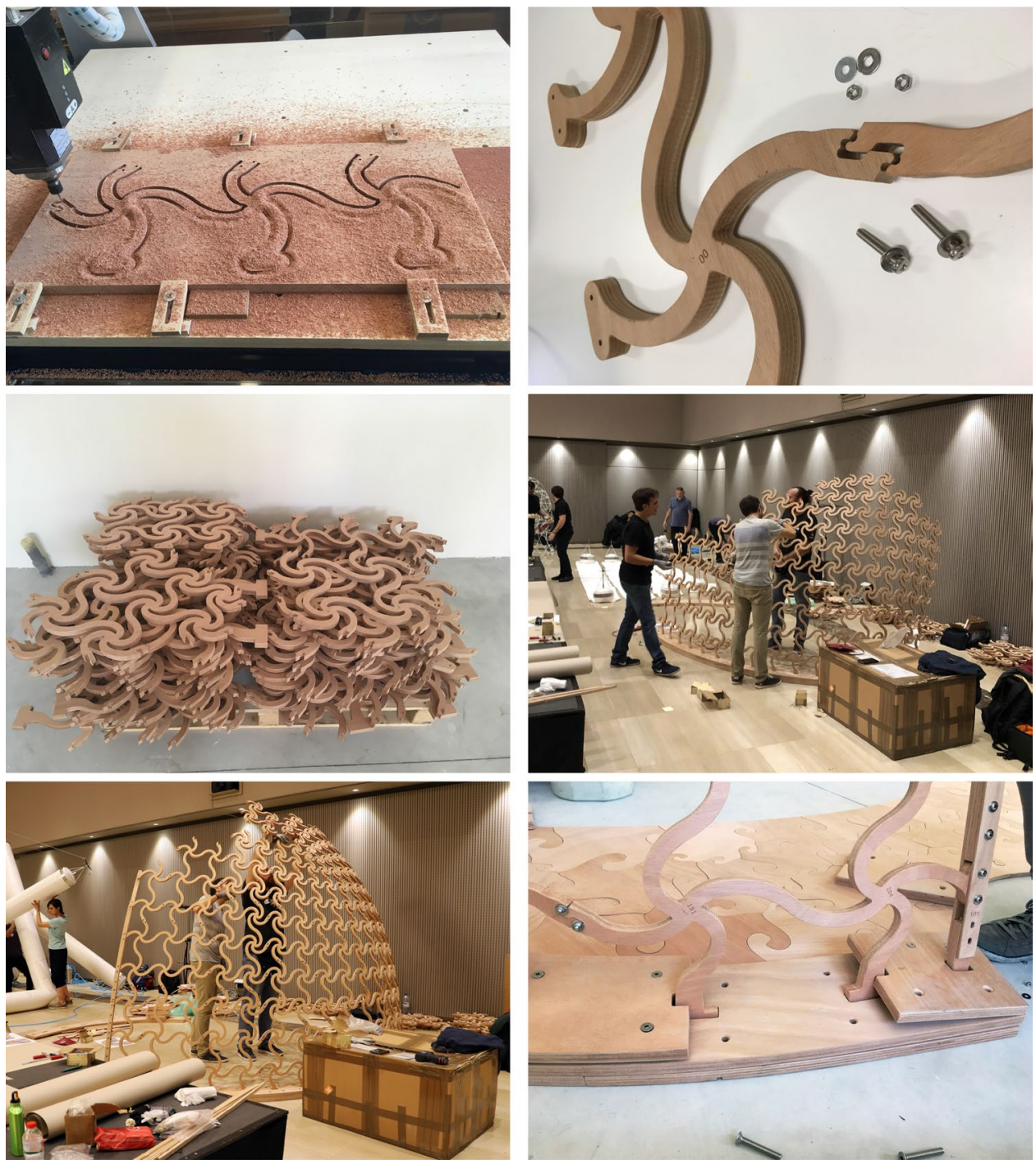

role in providing a restrain also during the assembly. Vertical supports provide additional help in this phase.

In order to avoid relying on rigid fixing, the Pavilion has been designed to be self-supporting. For this purpose, the lower patches are endowed with footings to be restrained in three-plies edge beams. These latter are linked and kept to the correct position by means of a plywood paving that has the role of balancing the thrust and twisting forces of the superstructure. The paving design is based on variabledensity Voronoi mesh with a size scaling from the supports to the center. The edges of the mesh polygons are dry interlocking. The final weight of the Pavilion is about $90 \mathrm{~kg}$, of which the spiral panes weight about $40 \mathrm{~kg}$.

\section{Conclusion}

This paper explores the possibility to extend the work FlexMaps [10] to the design of a free form plywood structure at a larger scale. This structure named FlexMaps Pavilion is bending-active and uses mesostructured panels shaped as spirals. The final shape is obtained by bending, progressively assembling and connecting the panels together. The structure results in a form-active shape, in which the initial stress induced by bending and twisting is used to shape panels that are initially flat. 
Fig. 9 Details of the FlexMaps Pavilion
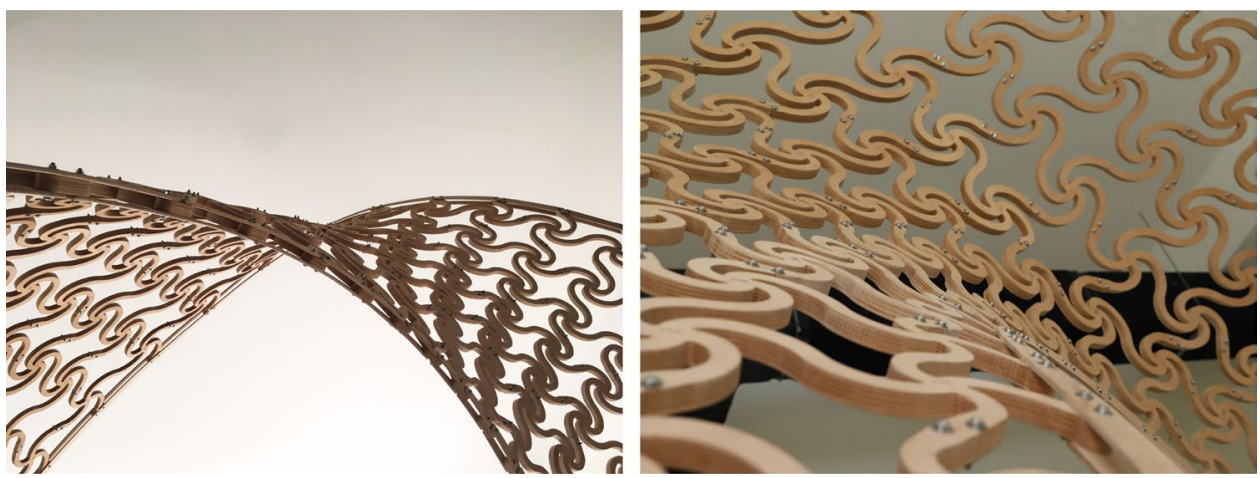

The FlexMaps algorithm is used to tile the twisted arch shape by means of spirals that are centered in a quadrilateral mesh. Then, the geometric parameters of the spirals are optimized through a data-driven method to be as close as possible to the target shape at the static equilibrium once assembled. Since this form-finding technique is computationally efficient but approximated, the obtained result has been validated through a finite element model in order to check the material stress and the expected deformation. At last, the panes are fabricated by CNC milling and assembled. To provide appropriate boundary conditions and self supporting capacity other elements are designed, such as the edge beams, the base and the paving.

The FlexMaps Pavilion proves the feasibility of extending at the architectural scale a technique initially designed for small scale objects. There are two main reasons that determine its feasibility. A first one is that a material commonly employed in the building industry such as plywood is suitable for the digital fabrication, and has mechanical properties that makes it compatible with the stress and displacement regimes required from this structure. A second one is that the patch-by-patch assembly is viable, it is compliant with statics of the panels and the structure throughout the stages of the construction, and can be performed by hand. This technique was unexplored before in architecture and is a novelty in the field of bending-active structures.

The major advantage of the form-finding approach provided by the FlexMaps algorithm is to match the curvature demands of the target shape. Thus, even complex non-developable shapes such as the selected twisted arch that are usually difficult to build in architecture become feasible (Fig. 9).

The FlexMaps Pavilion results in a lightweight bendingactive structure in which the initial stress is very low. Based on this experience, it can be stated that the FlexMaps concept applied to architecture appears very promising. However, the original pipeline [10] does not completely fit the purpose of generating larger-scale objects. There are fundamental additional steps that it would be useful to integrate in a new automated pipeline as further work, such as the finite element analysis and the generation of files-to-factory.

Using the FlexMaps technique does not pose any restriction on the target shape, but there is no degree of freedom on the selection of the tiling, which is fixed to be shaped as spirals, being the algorithm closely related to the specific geometric parameters and consequently to the pre-computed set of mesostructures. In the future, it could be interesting to investigate and test the applicability of different tiling geometries and their mechanical response.

Acknowledgements The FlexMaps Pavilion has been awarded First Prize at the "Competition and Exhibition of innovative lightweight structures" organized by the IASS Working Group 21 within the FORM and FORCE, joint international conference of IASS Symposium 2019 and Structural Membranes 2019 (Barcelona, 7-11 October 2019) with the following motivation: "for its structural innovation of bending-twisting system, connection constructability and exquisite craftmanship"[20]. The authors would like to acknowledge the Visual Computing Lab Staff of ISTI - CNR, in particular Thomas Alderighi, Marco Callieri, Paolo Pingi; Antonio Rizzo of IPCF - CNR; and the Administrative Staff of ISTI - CNR. This research was partially funded by the EU H2020 Programme EVOCATION: Advanced Visual and Geometric Computing for 3D Capture, Display, and Fabrication (grant no. 813170).

\section{Compliance with ethical standards}

Conflicts of interest The authors declare that they have no conflict of interest.

\section{References}

1. Lienhard J, Alpermann H, Gengnagel C, Knippers J (2013) Active bending, a review on structures where bending is used as a selfformation process. Int J Space Struct 28(3-4):187-196. https:// doi.org/10.1260/0266-3511.28.3-4.187

2. Lienhard J (2014) Bending-active structures: form-finding strategies using elastic deformation in static and kinematic systems and the structural potentials therein, $\mathrm{PhD}$ thesis, Institut für 
Tragkonstruktionen und Konstruktives Entwerfen (ITKE), Universität Stuttgart, p 36

3. Nicholas P, Tamke M (2013) Computational strategies for the architectural design of bending active structures. Int J Space Struct 28(3-4):215-228. https://doi. org/10.1260/0266-3511.28.3-4.215

4. Cuvilliers P, Yang JR, Coar L, Mueller C (2018) A comparison of two algorithms for the simulation of bending-active structures. Int J Space Struct 33(2):73-85. https://doi.org/10.1177/02663 51118779979

5. Gengnagel C, Hernández EL, Bäumer R (2013) Natural-fibrereinforced plastics in actively bent structures. Proc Inst Civ Eng Construct Mater 166(6):365-377. https://doi.org/10.1680/ coma.12.00026

6. Lienhard J, Knippers J (2015) Bending-active textile hybrids. J Int Assoc Shell Spatial Struct 56(1):37-48

7. Sonntag D, Bechert S, Knippers J (2017) Biomimetic timber shells made of bending-active segments. Int J Space Struct 32(3-4):149-159. https://doi.org/10.1177/0266351117746266

8. La Magna R (2017) Bending-active plates: strategies for the induction of curvature through the means of elastic bending of platebased structures, $\mathrm{PhD}$ thesis, Institut für Tragkonstruktionen und Konstruktives Entwerfen (ITKE), Universität Stuttgart, $\mathrm{p} 43$

9. La Magna R, Fragkia V, Längst $P$, Lienhard J, Noël R, Šinke Baranovskaya Y, Tamke M, Ramsgaard Thomsen M (2018) "Isoropia: an Encompassing Approach for the Design, Analysis and Form-Finding of Bending-Active Textile Hybrids," Proceedings of IASS Annual Symposia, vol. 2018, No. 15, pp. 1-8, International Association for Shell and Spatial Structures (IASS)

10. Malomo L, Pérez J, larussi E, Pietroni N, Miguel E, Cignoni P, Bickel B (2018) FlexMaps: computational design of flat flexible shells for shaping 3D objects. ACM Trans. Graph. 37(6):1-14. https:// doi.org/10.1145/3272127.3275076

11. Panetta J, Konaković-Luković MINA, Isvoranu F, Bouleau E, Pauly M (2019) X-Shells: A new class of deployable beam structures. ACM Trans. Graph. 38(4):1-15. https://doi.org/10.1145/33063 46.3323040
12. Laccone F, Malomo L, Péréz J, Pietroni N, Ponchio F, Bickel B, Cignoni P (2019) "FlexMaps Pavilion: a twisted arc made of mesostructured flat flexible panels," Proceedings of IASS Annual Symposia (Vol. 2019). International Association for Shell and Spatial Structures (IASS) pp 1-7(7)

13. Panetta J, Zhou Q, Malomo L, Pietroni N, Cignoni P, Zorin D (2015) Elastic textures for additive fabrication. ACM Trans. Graph. 34(4):135-1-135-12. https://doi.org/10.1145/2766937

14. Pérez J, Thomaszewski B, Coros S, Bickel B, Canabal JA, Sumner RW, Otaduy MA (2015) Design and fabrication of flexible rod meshes. ACM Trans. Graph. 34(4):1-12. https://doi. org/10.1145/2766998

15. Pietroni N, Puppo E, Marcias G, Scopigno R, Cignoni P (2016) Tracing field-coherent quad layouts. Comput Graph Forum Proc Pacific Graph. https://doi.org/10.1111/cgf.13045

16. Bommes D, Zimmer $H$, Kobbelt $L$ (2009) Mixed-integer quadrangulation. ACM Trans. Graph. 28(3):77. https://doi. org/10.1145/1531326.1531383

17. Pietroni N, Tonelli D, Puppo E, Froli M, Scopigno R, Cignoni $P$ (2015) Statics aware grid shells. Comput. Graph. Forum 34(2):627-641. https://doi.org/10.1111/cgf.12590

18. Kotelnikova-Weiler N, Douthe C, Hernandez EL, Baverel O, Gengnagel C, Caron JF (2013) Materials for actively-bent structures. Int J Space Struct 28(3-4):229-240. https://doi. org/10.1260/0266-3511.28.3-4.229

19. ANSYS ${ }^{\oplus}$. Academic Research Mechanical Release 18.0

20. IASS Working Group 21 (organizers),"Competition and Exhibition of innovative lightweight structures", at the FORM and FORCE, joint international conference of IASS Symposium 2019 and Structural Membranes 2019 (Barcelona, 7-11 October 2019): https://www.jjo33.com/iass-barcelona-2019

Publisher's Note Springer Nature remains neutral with regard to jurisdictional claims in published maps and institutional affiliations. 